\title{
Verifying Erlang Telecommunication Systems with the Process Algebra $\mu \mathrm{CRL}$
}

\author{
Qiang Guo ${ }^{1}$, John Derrick ${ }^{1}$, and Csaba Hoch ${ }^{2}$ \\ ${ }^{1}$ Department of Computer Science, \\ The University of Sheffield, \\ Regent Court, 211 Portobello, S1 4DP, UK \\ $\{$ Q.Guo, J.Derrick\}@dcs.shef.ac.uk \\ ${ }^{2}$ Faculty of Informatics \\ Eötvös Loránd Tudományegyetem \\ Pázmány Péter sétány 1/c., 1117 Budapest, Hungary \\ hoch@inf.elte.hu
}

\begin{abstract}
Verification is an important process in the development of Erlang systems. A recent strand of work has studied the verification of Erlang applications using the process algebra $\mu \mathrm{CRL}$. The general idea is that Erlang programs are translated into a $\mu \mathrm{CRL}$ specification, upon which the standard model checkers can be applied for checking the system's properties. In this paper, we pull together some of the existing work and investigate the verification of an Erlang telecommunication system in $\mu \mathrm{CRL}$. This case study uses a server-client structure and incorporates timing restrictions and is designed and implemented using a number of Erlang/OTP components. We show how this system is translated into a $\mu \mathrm{CRL}$ specification by using the defined rules, after which system properties are checked via the toolset CADP. Through studying the verification of such an application, we aim to validate the effectiveness of the translation rules in an integrated way.
\end{abstract}

Keywords: Erlang, Telecoms case study, Process Algebras, $\mu$ CRL, Translation, Verification.

\section{Introduction}

Erlang [1 is a concurrent functional programming language with explicit support for real-time and fault-tolerant distributed systems. It is available under an Open Source Licence from Ericsson, and since its conception its use and development has widened to a number of sectors such as TCP/IP programming, etc.

A key feature of Erlang is the Open Telecom Platform (OTP) architecture where generic components are encapsulated as design patterns, each of which solves a particular class of problem. These patterns include servers, supervisors, finite state machines etc. This makes Erlang an ideal programming language for the development of fault-tolerant systems containing soft real-time requirements.

Verification is an important part of the Erlang system process. Although Erlang has many high-level features, verification can be still non-trivial. A number 
of possible approaches have been explored, including the one we investigate here: abstract an Erlang application into a formal model, upon which model checking 9. techniques can be applied. This approach has recently been applied to the verification of Erlang programs and OTP components 235771416] where the process algebra $\mu \mathrm{CRL}$ [13] has been used as the formal language upon which verification is carried out.

Arts et al. 223 initiated this strand of work and proposed rules for translating Erlang syntax and the OTP components gen_server, supervisor into $\mu$ CRL. Benac-Earle [5] continued with the work and developed a toolset, etomcrl, to automate the process of translation. Guo et al. extended the work by proposing a model for the translation of the OTP finite state machine gen_fsm [14 and defining rules for coping with Erlang timeout events in $\mu \mathrm{CRL}$ [16.

However, rules for the translation of OTP gen_server, supervisor, gen_fsm and Erlang timeout have, so far, only been independently evaluated via some small examples, and no work has evaluated these rules in an application where the above components are integrated as a system. One might argue that if all rules are applied in an integrated way, will they show the similar effects for system verification as they demonstrated in the existing work? Moreover, will a state space explosion mean that effective verification is lost?

In this paper, we attempt to look at these questions by investigating the verification of an Erlang telecommunication system in $\mu$ CRL. A telecommunication system of server-client structure and timing restriction for operation is developed with Erlang/OTP. The system integrates the use of the supervisor, gen_server, gen_fsm components and uses explicit timeout events. We show how the system is then translated into a $\mu \mathrm{CRL}$ specification using the proposed translation rules. We then verify a number of system's properties by using the model checker CADP [8] and investigate the changes of state space when the number of clients increases. The experimental results suggest that when being applied in an integrated system, the translation rules show the similar effect for system verification as being applied independently.

The paper is organized as follows: Section 2 describes a telecommunication system that is used as a case study in this paper; Section 3 implements the system with Erlang programming langauge; Section 4 discusses the translation of the telecoms case study into $\mu \mathrm{CRL}$; Section 5 looks at the system verification using the standard model checker CADP; conclusions are drawn in Section 6.

\section{Telecommunication System}

In this section we give an overview of our case study, which is implemented in Erlang in Section 3 .

\subsection{System Infrastructure}

Our telecoms case study uses a client-server structure, and comprises of a database server (DBS) that is used to maintain all client's data and a number of 
functional servers (FS) that will process clients' requests. An FS has a capacity and a user list. The capacity defines the maximum number of clients (mobiles) that can be connected to a server, while, the user list saves all clients (mobiles) that have been connected to this server. The telecoms system illustrated in Figure 1 is designed with one DBS (named as $D B$ ), three FSs (named as $S V R_{-} 1$, $S V R \_2$ and $S V R \_3$ ) and five clients (named as $M_{-} 1, M_{\_} 2, M_{\_} 3, M_{-} 4$ and $M_{\_} 5$ ).

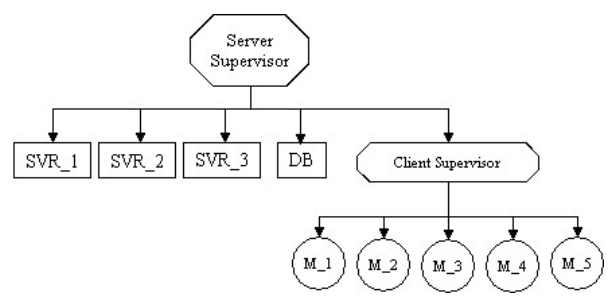

Fig. 1. Telecoms system designed with one DB, three FSs and five clients

Once the system starts up, an FS can communicate with DBS and any other FSs. A client can communicate with any FSs, and can perform some functional operations such as calling and top-up. The behaviour of clients is described in section 2.2. Each client has an account maintained in the DBS, and in order to make a phone call, a client needs to save enough money in its account.

Before performing any functional operations, a client needs to connect to an FS. After being connected to an FS, the client's identity is maintained in the FS' user list. A client can only be connected to one FS, and if a client has connected to an FS and tries to connect to another FS, an error message will be returned and the request is denied. When a client disconnects, the appropriate FS cuts off the connection and removes this client from the user list to release the resource. The FS will notify all other FSs about the changes of its clients' state so that they can correctly respond to the client's requests.

\subsection{Client Behaviour Modelling}

The behaviour of a client (mobile) is modeled as a finite state machine (FSM), and the initial design is shown in Figure 2. There are four states: idle, connected, calling and top_up, where initially, the system is set to the idle state.

The FSM defines the behaviour of a number of operations: connecting, disconnecting, calling, terminating, top_up and cancelling. Before performing any operations, a client FSM needs to connect to an FS through sending the connecting request. If the FS replies $\{$ ok, connected $\}$, it indicates that the request is accepted and the connection is set up. The FSM moves to the state connected; otherwise, if $\{$ error,busy\} arrives, it suggests that the server has reached it maximum capacity. The client will send the connecting request to another FS. The 


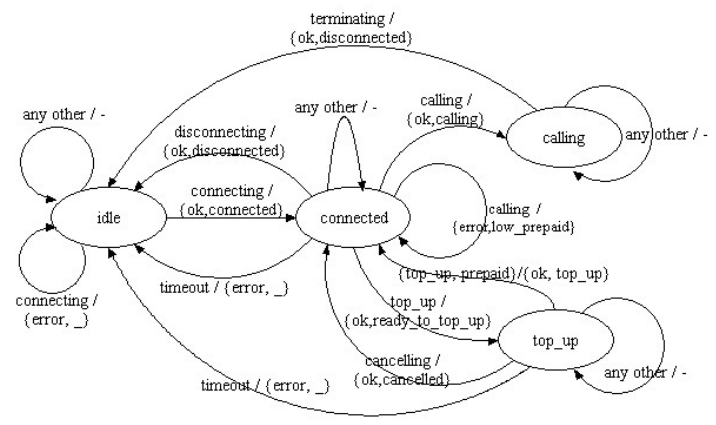

Fig. 2. Client behaviour modelled as an FSM

FSM remains in the state idle. The client will iteratively send the connecting request to each FS until the connection is approved and set up by an FS.

A client can stop the connection by sending the disconnecting request to the FS. Once the disconnecting request being received, the FS will cut off the connection and remove the client from its user list to release the resource. A reply message $\{$ ok, disconnected $\}$ will be sent to the client, and upon receiving this reply, the FSM will be reset to the state idle.

When in the state connected, a client can make a phone call through calling operation or top up it account through top_up operation. When the calling request is sent, if its account has enough money, a client will receive $\{$ ok, calling $\}$ from the appropriate FS, enabling the calling process. The FSM then moves to the state calling; otherwise, \{error, low_prepaid $\}$ will be received, asking the client to top up its account. The FSM remains in the state connected.

When in the state calling, only the terminating operation can terminate a calling process. This prevents the calling process from being disrupted by some unintended actions. When the terminating request is sent, the DBS will reduce the amount of money from this client's account. The FS then cuts off the client's connection and releases the resource from its user list. Meanwhile, a message $\{\mathrm{ok}$, disconnected $\}$ is sent to the client, and on receiving the reply, the FSM is reset to the state idle.

After being connected to an FS, a client can ask to top up its account by sending the top_up request. If $\{o k$, ready_to_top_up $\}$ is received, it indicates that the top up process is accepted by the FS, and the FSM moves to the state top_up. The client can then transfer money to its account through $\left\{t_{0} \_u p\right.$, Prepaid $\}$ operation where Prepaid is the amount of money that is about to be transfered. When the transaction succeeds, the FS replies the client with $\left\{o k, t_{o} p_{-} u p\right\}$ and when receiving such a reply, the FSM returns to the state connected.

A client FSM has a timing restriction applicable when in states connected or top_up. Specifically, when the FSM is directed to the state connected or top_up, a timer will be instantiated which enables the timing process. If, within the predefined time period, no action is performed by the client, a timeout event will be generated and sent to the FS. By receiving timeout event, the FS cuts 
off the connection and releases the resource from its user list. The FSM is then reset to the state idle.

\section{Erlang Implementation}

Erlang is used to implement the telecoms system, making use of the OTP design patterns as is common practice.

\subsection{Functional Server Implementation}

The functional server (FS) is implemented using the Erlang/OTP gen_server module. A generic server is implemented by providing a callback module where (callback) functions are defined specifying the concrete actions of the server such as server state handling and response to messages.

In this work, the callback function handle_call in the FS module is comprised of two parts. One (using keyword request) processes client's requests; the other (using keyword notify) deals with the notification sent from FSs:

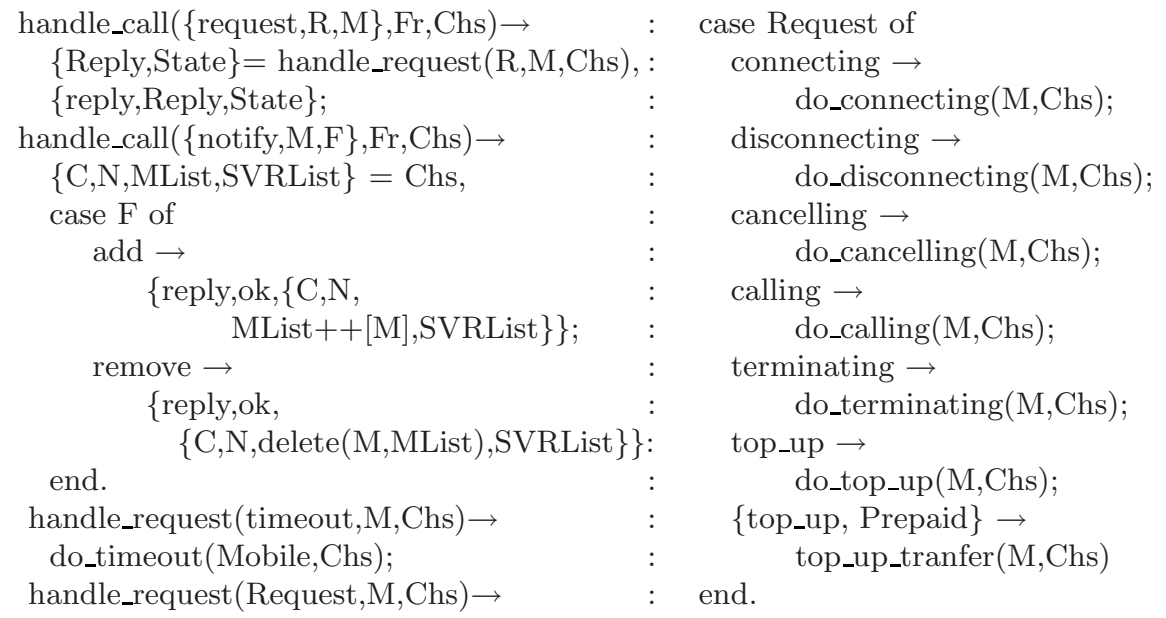

The internal variable $C h s$ (defined by the Erlang system for saving values) is defined with the form of $\{C, N, M L i s t, S V R L i s t\}$ where $C$ defines the FS' capacity, $N$ counts for the number of clients that has been connected to this FS, MList saves clients that have connected to this FS and SVRList saves all FS servers running in the system.

The function handle_request(Request, Mobile, Chs) is defined where a list of do functions is called to process client's requests. The function do_connecting is defined to set up connection between the FS and a client. It first examines whether the FS reaches its maximum capacity. If the FS is full, \{error, busy $\}$ will be returned to the client; otherwise, the connection is set up. The client is then registered in MList and $N$ is increased by 1 . The function do_disconnecting is defined to disconnect a client from the FS. When the disconnecting request arrives, the FS cuts off the connection and then, by calling the function notify_servers, notifies other FSs (saved in SVRList) to release the resource (removes the client from MList). 
The do_calling function monitors the calling process. When the calling request is received, the function reads the client's data from the DBS and checks whether it has enough money for making a call. If so, the calling process is enabled; otherwise, $\{$ error, low_prepaid $\}$ is replied, asking the client to top up its account. When the client finishes calling, it sends the terminating request to the FS. Upon receiving the request, the FS enables the do_terminating function to subtract amount of money from the client's account, and then cut off the connection through disconnecting operation. The function do_top_up and top_up_tranfer are defined to top up client's account. When the top_up request is received, the FS enables the process by replying the client with $\left\{o k, r e a d y_{-} t o_{-} t o p_{-} u p\right\}$. Once the client's money is received, the top_up_tranfer function is enabled to complete the transaction.

\subsection{Client Implementation}

The client behaviour is implemented using the OTP gen_fsm module, and the state transition rules are defined conforming to the following convention:

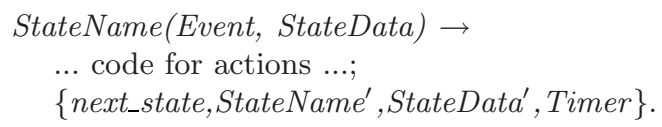

where the state function returns a tuple that contains the name of the next state, StateName', and an updated state data, StateData'. Timer is an optional element, if it is set to a value, a timer is instantiated, and a timeout event will be generated when the time-up occurs. The function send_event is defined to trigger a transition. When send_event is executed, the gen_fsm module automatically calls the current state function.

In accordance with the design given above, four state functions are defined in the client module: idle, connected, calling and top_up. The state function idle initiates a connecting request to the FS $S V R$. If the FS $S V R$ replies the FSM with $\{$ ok, connected\}, the request is accepted and the connection is set up. The FSM moves to the state connected; otherwise, the request is denied and the FSM remains unchanged.

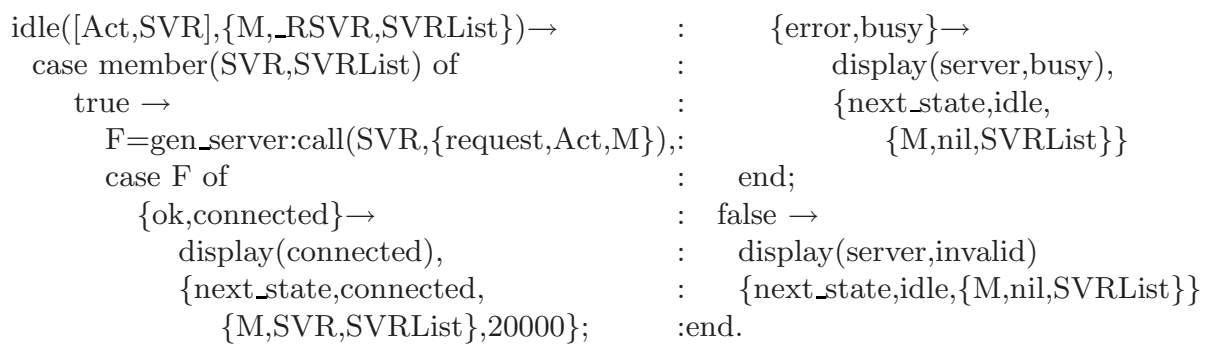

Once the client is connected to an FS, an event will trigger the state function connected, which evaluates the request and then makes decisions for the consequent actions. For example, if a calling request is made, the function will call the FS to evaluate the client's state. If the client has enough money in its account, $\{$ ok, calling $\}$ will be returned to approve the calling process, and upon receiving the reply, the FSM moves to the state calling. 


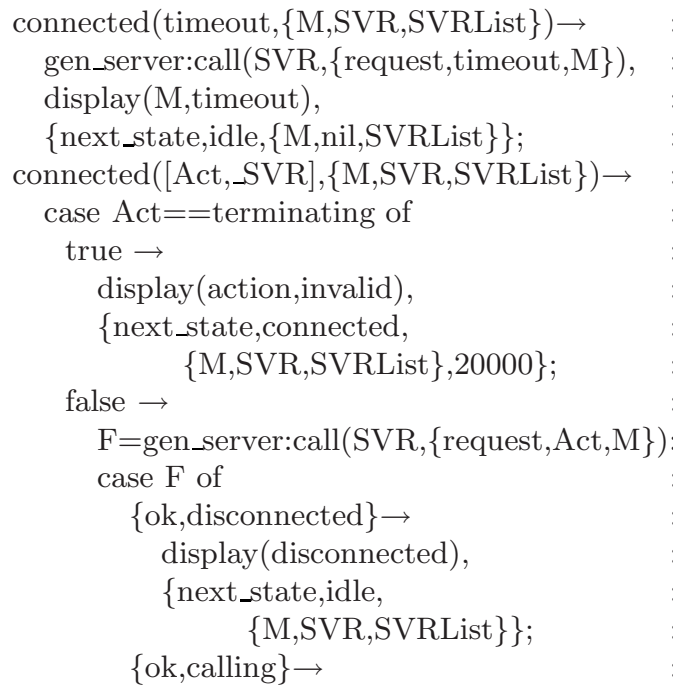

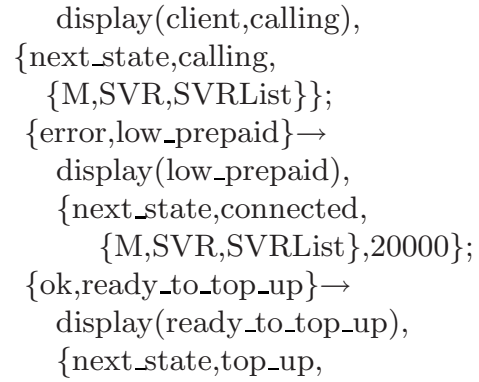

$\{$ M,SVR,SVRList $\}, 20000\}$;

-Other $\rightarrow$

display(action,invalid), next_state,connected,

$\{$ M,SVR,SVRList $\}, 20000\}$ end end.

When in the state calling, only the terminating action can stop the calling process. This prevents the calling process from being disrupted by any unintended actions.

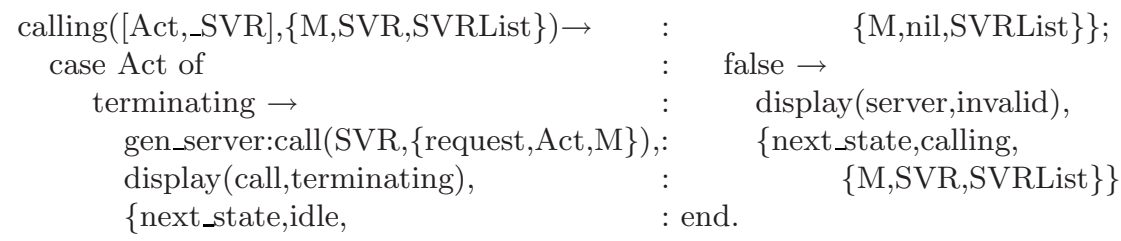

false $\rightarrow$ M,nil,SVRList $\}$

display(server,invalid),

$\{$ next_state,calling, $\{$ M,SVR,SVRList $\}$ \}

When being connected to an FS, the client can ask to top up its account by sending the top_up request to the FS. If $\left\{o k, r e a d y_{-} t_{-} t_{o} p_{-} u p\right\}$ is replied, the top up process is enabled, and the FSM moves to the state top_up. An action will trigger the state function top_up to either start the transaction by $\left\{t_{\text {_ }} p_{\_} u p\right.$, Prepaid $\}$ operation (Prepaid is the amount of money the client is about to transfer), or cancel the process by sending the cancelling request.

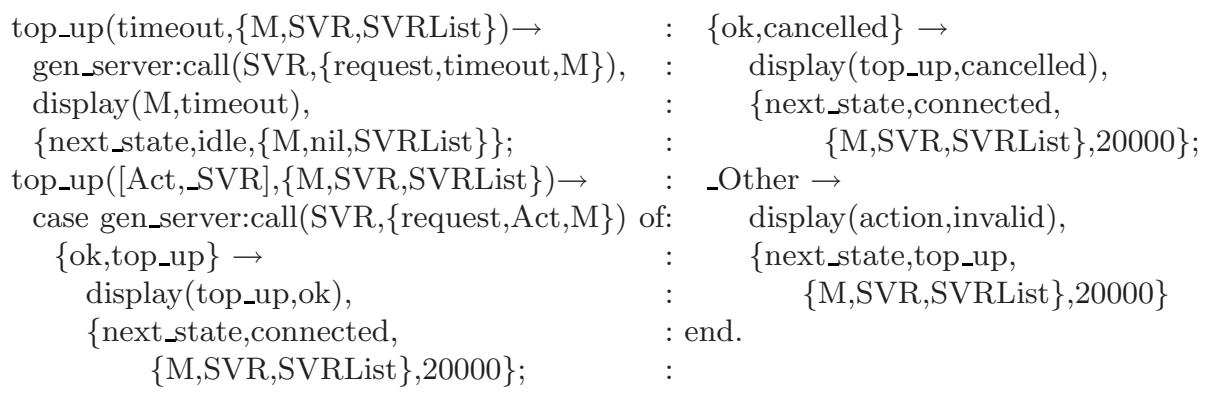

When the FSM moves to the state connected and top_up, a timer is initiated. The timer is set to $20,000 \mathrm{~ms}$. If within the time period, no action is performed, a timeout event will be generated and sent to the FS. The FSM is reset to the state idle. A function command is defined to simulate the receiving of external actions. It calls gen_server:send_event to triggers the state functions. 


\section{Translating Our Case Study into $\mu$ CRL}

In this section we describe the verification methodology used in this project. It uses the process algebra $\mu \mathrm{CRL}$ (micro Common Representation Language) [13] which is an extension of the process algebra ACP [4, where equational abstract data types have been integrated into the process specification to enable the specification of both data and process behaviour. We assume the reader is familiar with $\mu \mathrm{CRL}$.

\subsection{Pre-processing}

Before the translation begins, the Erlang input is pre-processed which transforms the Erlang code into an optimized format, but has identical behaviour. For example, Erlang makes extensive use of pattern matching in its function definitions, and overlapping between patterns could lead to the system being represented by a faulty model in $\mu$ CRL. This work transforms Erlang programs using the techniques discussed in 15 where pattern matching clauses in a function are replaced with a series of calling functions, each of which being guarded by the function patterns_match.

For example, the function handle_request is transformed as shown above. A data structure, called a Structure Splitting Tree (SST) [15], is applied for pattern evaluation, and the use of such an SST for pattern evaluation guarantees the elimination of overlapping between patterns in the transformed program.

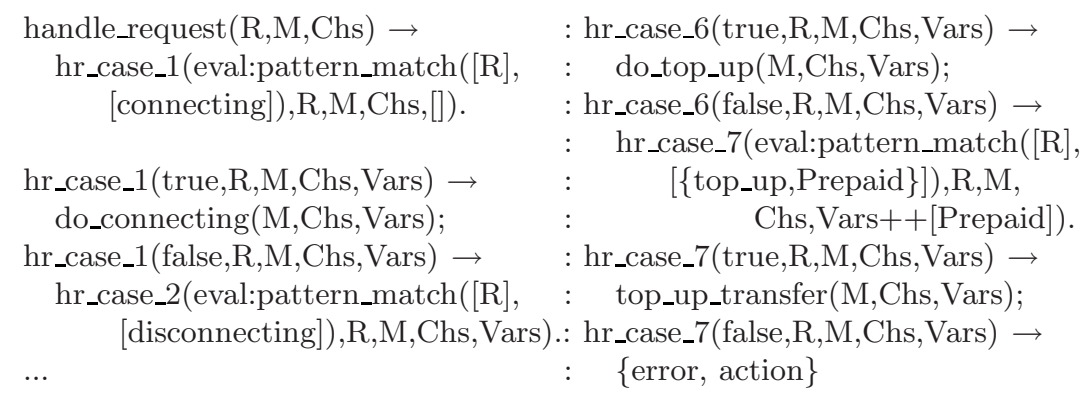

\subsection{Translating the Server Component}

Erlang performs synchronous and asynchronous communications using the generic server primitives gen_sever:call / handle_call and gen_sever:cast / handle_cast respectively. One then has to model both synchronous and a synchronous communication in $\mu$ CRL, and to do so we use a Server_Buffer process (described in [5]). A data type GSBuffer is defined to contain the data for the process Server_Buffer. The actions gen_server_call and gscall are defined to write a message to the buffer while, gshall and handle_call to read a message from the buffer.

The database server is translated into a process $S D B$, and it maintains a number of clients, each in the form $\{$ CName,Prepaid,State $\}$ where CName is the client's name, Prepaid shows the amount of money saved in the client's account and State indicates whether the client is connected to an FS or not. The actions server_read_db and $d b \_s e n d \_d a t a$ are defined to read a client's data out from the process, server_read_db $\mid d b_{\_} s e n d \_d a t a=r e a d \_d b$. The actions servr_update_db and $d b_{-} a c k \_r e q u e s t$ are used to update the client's data in the process, servr_update_db $\mid d b_{-} a c k_{-} r e q u e s t=u p d a t e_{-} d b$. 
The functional server (FS) is translated into a process server. The process contains a server ID $S V R I D$, a capacity $C$ and a client list $C L s$. It uses the action handle_call to receive the client's requests: when a request is received, the server process first checks whether the request is made for itself. If so, the process calls the process handle_request to tackle the request; otherwise, its returns without changing anything.

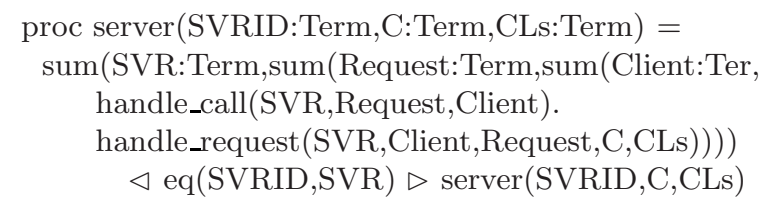

For each client's request, a request process is defined. Once the handle_request is enabled, it selects the corresponding request process. The selected process first performs all pre-defined actions and then replies the client with a message through the action gen_server_reply. For example, when the connecting request is received, the process handle_request_ connecting is activated. It first checks whether the server reaches its maximum capacity. If the server is full, the process goes back to the process server without changing anything; otherwise, if the client has not been connected to a server before, the process sets up the connection and replies the client with $\{$ ok, connected $\}$.

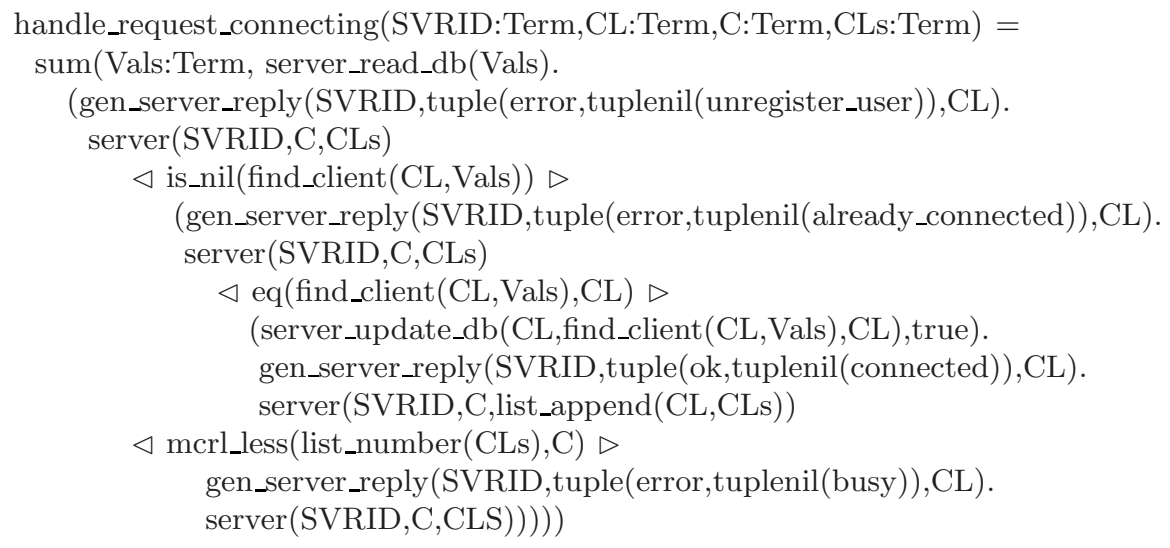

The process handle_timeout is defined to deal with timeout event. Once the timeout event is generated from a client, the process handle_timeout will be activated. It cuts off the connection between the server and the client and removes the client from its user list to release the resource.

\subsection{Translating the Client Component}

The client was initially modelled as an FSM and implemented using the OTP gen_fsm. This is then translated into $\mu \mathrm{CRL}$ using techniques defined in 14, where the translation process is comprised of two parts, simulating state management (SSM) and state function translation (SFT).

In this work, a (one place) stack is used to perform the SSM which is modified by using a global variable $(\mathrm{GV})$ process. A GV process contains a list of indexed GVs, where each $\mathrm{GV}$ is of the format $\{$ VName, Val $\}$ where the VName gives the variable's name and the $V a l$ the value. A GV process with three GVs, $V_{1}, V_{2}$ and $V_{3}$, is defined as follows: 


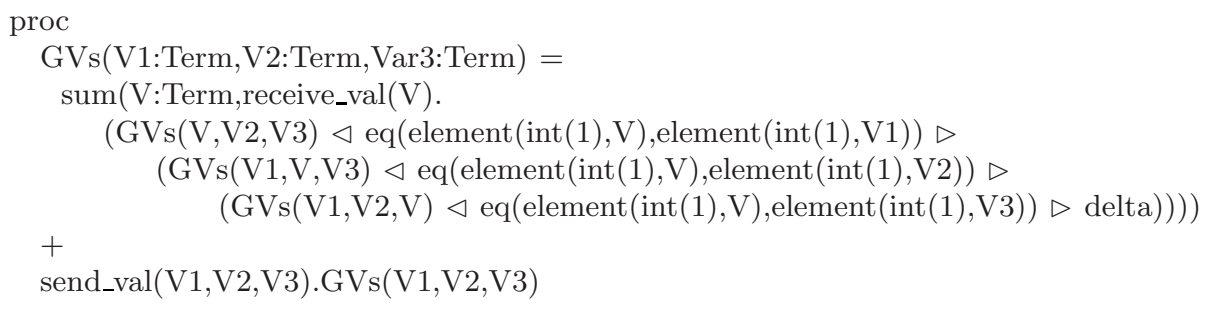

A GV can be read out through the actions read_val send_val, write_val $\mid$ receive_val= write, and be modified through the actions write_val / receive_val, write_val | receive_val = write. We use a GV to stand for a client where VName and Val are used to save the client FSM's current state and the state data respectively. We found that, by applying such a modification, the state space is largely reduced.

The process receive_cmd is defined to receive commands generated from the external actions. For each client, a unique ID CLID is associated with all its FSM processes. Once a command is received, the process receive_cmd calls the process read_clients. According to the CLID, the designated FSM's current state and the state data are read out. The corresponding state process is then selected for performing all defined actions. Once the execution of the state process finishes, the FSM moves to the process fsm_update_state to update the current state and the state data.

For example, when the FSM is in the state idle and the connecting command is received, the state process $f s m_{-} i d l e$ is activated. The process fsm_idle sends the request to an FS and then waits for reply. If the FS returns busy, the process will calls for another FS; otherwise, the connection is set up. The process then calls for the process fsm_update_state to update the state connected as the current state. Thus we have the following:

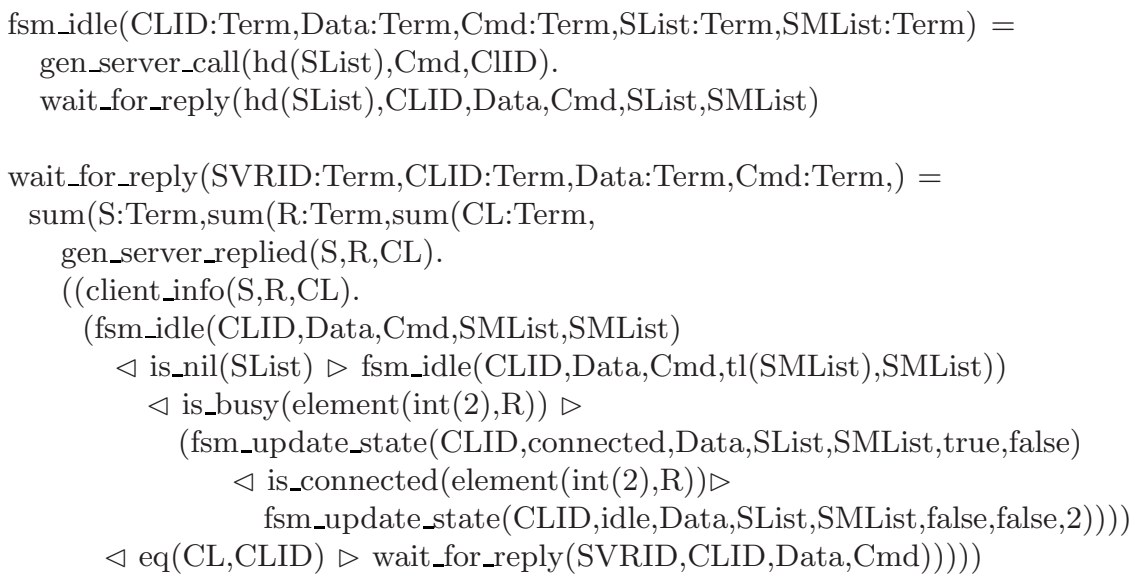

The process fsm_update_state is parameterized with two arguments, FT and FTM. The FT determines whether the updated current state has timing restrictions on it; the FTM decides whether the process will be terminated due to some unexpected events. If the newly updated current state process has timing restrictions, the $F T$ will be set to true, which enables the process fsm_timing to count down the time. If, within the predefined time period, no external action is performed, a timeout event will be 
generated and sent to the FS. Afterwards, the process is terminated by setting the FTM to true. Thus we have the following output from the translation process:

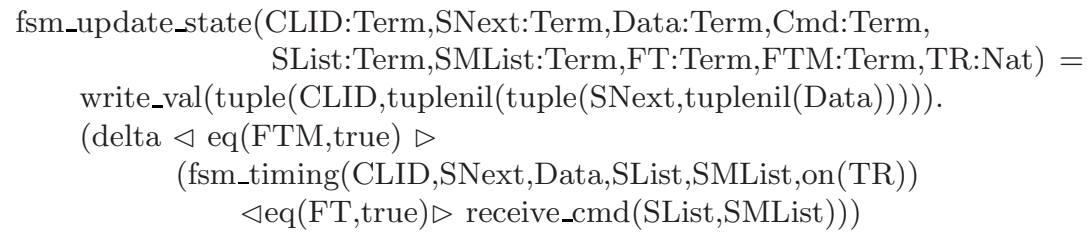

The process fsm_timing and the process count_down are parameterized with a timer 16. By using an explicit tick action in the process count_down, we apply a discrete-time timing model to support the translation of timeout event. When the process fsm_timing is called at the first time, the timer $t$ is initiated and initialized. The process will either call for the process count_down to start the timing process or the receive_cmd process to continue with another external command.

fsm_timing(CLID:Term,SNext:Term,Data:Term,SList:Term,SMList:Term,t:Timer) = count_down(CLID,SNext,Data,SList,t) + receive_cmd(SList,SMList)

When the count_down process is activated, it checks whether the timer expires (using the function expire(t:Timer)). If not, the process will first perform the tick action once, standing for the passing of one time unit. The process then moves back to the process fsm_timing, counting down the timer $t$ by one unit $(\operatorname{pred}(t))$; otherwise, if the timer expires, the process $f_{s m \_}$update_state is called, with the next state $S N e x t$ being reset to idle and the FTM to true.

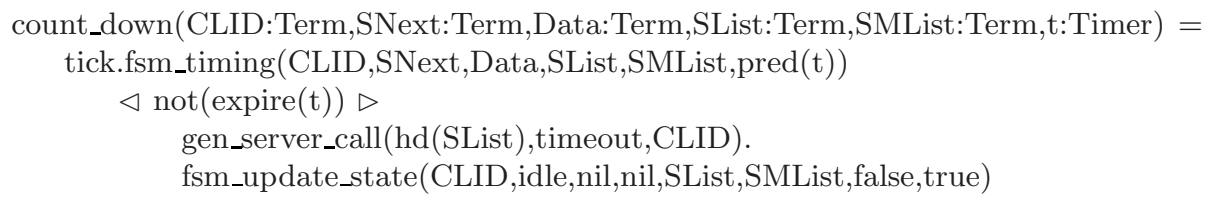

\subsection{System Translation}

By considering the translation of server section and client section together, the system is translated into a complete $\mu \mathrm{CRL}$ specification. In the specification, every server and client are initialized with a unique client ID. For each client, the process client_cmds(CLID, CmdList) is applied to initialize a list of external actions where CLID indicates the client's ID and $C m d L i s t$ saves the sequence of commands. A client receives a command through the action $r_{-} c m d(C L I D, C m d)$.

A client sends a request to an FS through the action gen_server_call(SVRID,Cmd, $C L I D)$ where $S V R I D$ indicates the target server ID while $C L I D$ the sender's ID. A client receives a reply through the action gen_server_replied(SVRID, Reply,CLID) where $S V R I D$ shows from which server the reply comes and CLID indicates to which client the reply is sent.

When receiving a request, an FS process compares its ID with the received SVRID to examine whether the request is made for the server itself. If so, the request is accepted and the consequential actions will be performed; otherwise, the FS process ignores the request and returns without changing anything. Similarly, when receiving a reply, a client process compares its ID with the received CLID to check whether the message 
is replied to the client itself. If so, the client process performs the actions extracted from the reply; otherwise, the process ignores the message. Through ID checking, a peer-to-peer communication structure is defined in the $\mu$ CRL specification.

We have now reached a point whereby the design, as implemented in Erlang/OTP has been translated (in fact, abstracted) to a $\mu$ CRL specification, and we now described how properties of the initial design can be checked on this model.

\section{$5 \quad$ Verifying the Telecommunication System with $\mu$ CRL}

In this section, a number of system properties are abstracted and verified. In our experiments, the property under verification (PUV) is devised in a way where the behaviour of FS(s), the behaviour of client(s) and the communication between the FS and the client are considered as an integrated whole. Thus, instead of focusing on particular individual components, the properties we are concerned with in this case study are defined across the whole system.

\subsection{Property Verification}

The system used for simulation is constructed as shown in Figure 1 where three functional servers $\left(s v r \_1, s v r \_2\right.$ and $\left.s v r \_3\right)$ and five clients $\left(m \_1, m \_2, m \_3, m \_4\right.$ and $\left.m \_5\right)$ are used. We initialize the capacity of every server to 1 . The clients $m_{-} 1, m_{-} 3, m_{-} 5$ are preset with $£ 1$ in their accounts, while $m_{-} 2, m_{-} 4$ with $£ 0$. We define that the minimum cost of making a phone call to be $£ 1$. The timer for the functions with timing restriction is set to $20,000 \mathrm{~ms}$, and we define the passing of one time unit as $10,000 \mathrm{~ms}$, represented by one tick action. As discussed in Section 3.2 the gen_fsm:send_event is often called through external actions. Therefore, before starting a simulation process, for each client FSM, a sequence of actions needs to be initialized in the process Client_Cmds to simulate the external behaviour.

We first devise two experiments to verify the system's client-server property. In the first experiment, the client $m_{-} 1$ attempts to make a phone call while $m_{-} 2, m_{-} 3, m_{-} 4$ and $m_{-} 5$ are idle; in the second, the client $m_{-} 2$ tries to make a phone call while $m_{-} 1$, $m_{-} 3, m_{-} 4$ and $m_{-} 5$ are idle. Thus, for both these initial experiments, only one client is active. Through these two experiments we want to check whether the FS(s) and the client act as defined in design, and whether the communication between the FS and the client is correctly running.

The commands for the two experiments are coded in the list $C m d=$ cons(connecting, cons(calling, nil)) and initialized in the process client_cmds respectively. The Labelled Transition Systems (LTSs) derived from the toolset CADP 8] are shown in Figure 3 and 4 Here, we hide the actions update_db and read_db as internal actions, denoted by $i$ in the LTSs.

Verification of the properties can be performed by using the model checker CADP, where the system properties are formalized by a set of temporal logic formulae. For example, in the first experiment, to check "without being connected to svr_1, $m_{-} 1$ cannot make a phone call.". This property can be formalized as:

$$
\left.\left.\left[\text { not(client_info }\left(m_{-} 1, \text { connected, svr_1 }\right)\right)^{*} \text {. client_info }\left(m_{-} 1 \text {, calling, svr_1 }\right)\right)\right] \text { false }
$$

Similarly, to check "when $m_{-} 1$ is connected to $s v r_{\_} 1$, without delaying enough time (two tick actions being consecutively performed), a timeout event cannot be generated.", the property is formalized as: 


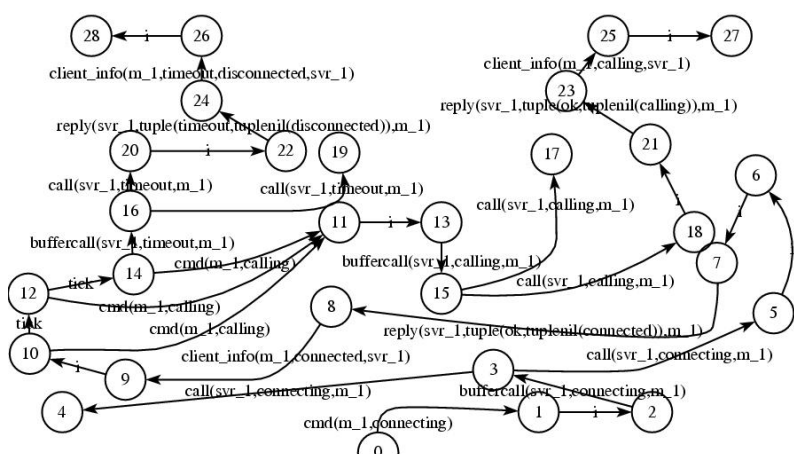

(0)

Fig. 3. LTS: The client $m_{-} 1$ makes a phone call

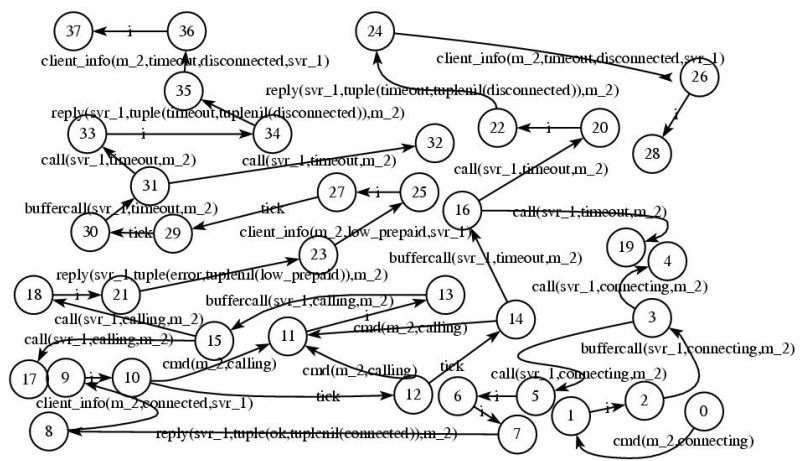

Fig. 4. LTS: The client $m_{-} 2$ makes a phone call

[true*. client_info $\left.\left(m_{-} 1 \text {, connected, svr_1 }\right)^{*}\right]$

$<\operatorname{not}(\text { 'tick.tick') })^{*}$. client_info(m_1, timeout, disconnected, svr_1) $>$ false

In the second experiment, to check "when $m_{\_} 2$ is connected to $s v r_{-} 1$, if $m_{-} 2$ has not preset enough money in its account, the calling process cannot be accepted.", the property is formalized as:

$<$ true* . client_info(m_1, connected, svr_1 $)^{*}$. client_info $\left(m_{-} 2 \text {, low_prepaid, svr_1 }\right)^{*}$. client_info $\left(m_{-} 1\right.$, calling, svr_1 $)>$ false

Next, we construct an experiment to examine the system's behaviour where more than one clients are active. Two clients $m_{-} 1$ and $m \_2$ request to connect to a server simultaneously. Since the capacity of the FS is set to 1, according to the design, when an FS, for example svr_1, accepts the request of a client, say $m_{-} 1$, it should reply the other $m_{\_} 2$ with busy; the client $m_{2} 2$ should afterwards request a connection to svr_2. Similar to the previous experiments, we want to check the behaviour of FSs and the clients in an integrated way, but use more complicated system structure. Figure 5 illustrates the derived LTS. Here, the actions call, buffercall and reply are hidden as internal actions as well. 


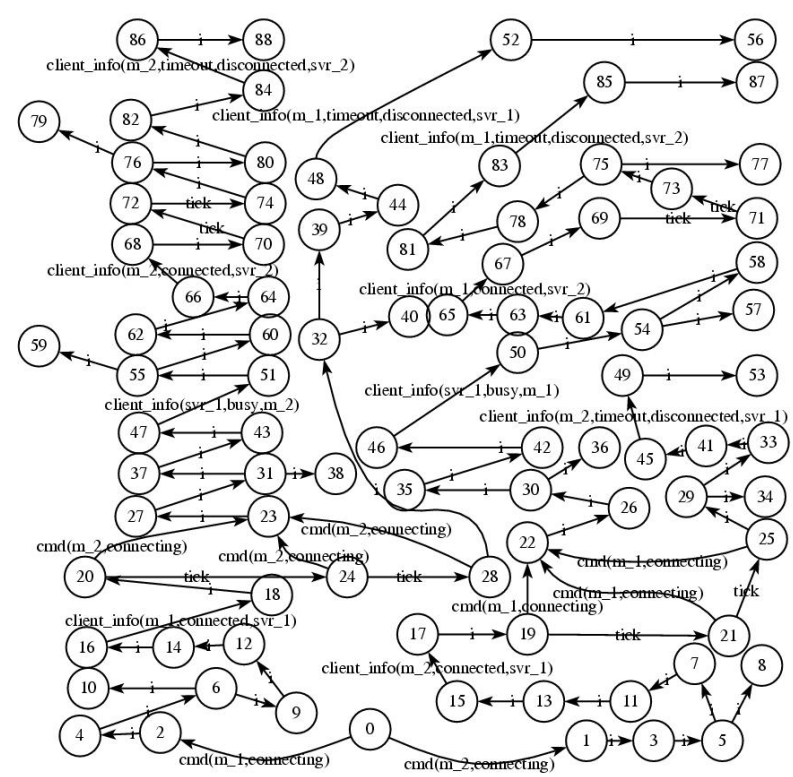

Fig. 5. $m_{-} 1$ and $m_{-} 2$ make requests to connect

A number of properties can then be automatically verified via CADP. For example, to check "when $m_{-} 1$ is connected to $s v r_{-} 1$ and $m_{-} 2$ requests to $s v r_{-} 1, s v r_{-} 1$ will reply $m \_2$ with busy.". The property is formalized as:

$<$ true*. client_info $\left(m_{-} 1\right.$, connected, svr_1 $) *$. cmd $\left(m_{\_} 2\right.$, connecting $) *$. client_info $\left(m \_2\right.$, busy, svr_1 $)>$ true

Another property we want to check is formalized as:

$<$ true* $^{*}$ cmd $\left(m \_2\right.$, connecting $) *$. client_info $\left(m \_2\right.$, busy, svr_1) *.

$\mathrm{cmd}\left(m_{-} 2\right.$, connecting $) *$. client_info $\left(m_{-} 2\right.$, connected, svr_2 $)>$ true

stating that "when $m_{\_} 2$ requests to connect to $s v r_{-} 1$ and receives the reply of busy, it will request to connect to $s v r_{\_} 2$ and its request will be accepted by $s v r_{\_} 2 . "$

We also devise an experiment to show how the methodology can be used for fault detection. A system with two FSs $\left(s v r \_1\right.$ and $\left.s v r \_2\right)$ and four clients $\left(m \_1, m \_2, m_{-} 3\right.$ and $m_{-} 4$ ) is constructed, where four clients simultaneously request a connection to an FS. Both $s v r_{-} 1$ and $s v r_{\_} 2$ are meant to be designed with a capacity of 2 , and we assume that one (say svr_2) by mistakenly implemented with a capacity of 1 . This could cause serious problems as one client will iteratively make a request to connect to the system without knowing whether he/she will ever get through.

The erroneous implementation is then translated into a $\mu \mathrm{CRL}$ specification from which we derive its LTS, however, since it has a total of 354 states and 407 transitions it cannot be clearly presented here. As usual we use the toolset CADP to verify the properties.

One way to detect such a problem is to check whether the four clients are successfully connected to the FSs. Since the system is designed with the capacity of 4 , all four clients should have connected to an FS. Thus, for each client, we define the following property: 
[true*. "cmd( $m_{-} i$, connecting)" *]

(<true* "client_info $\left(m_{\_} i\right.$, connected, svr_1) )" $>$ or

$<$ true* "client_info $\left(m_{-} i\right.$, connected, svr_2 $\left.)\right)$ " $>$ ) true

stating "when client $m_{-} i$ sends connecting request to the system, its request should be either accepted by sver_1 or by sver_2". Using these properties, the CADP model checker can correctly distinguish the correct and faulty implementations based upon the design we wish to check against.

\subsection{State Space Investigation}

In addition to system wide property checking, we were interested in whether the integrated system had a tractable state space as the size of its components grew, thus we also investigated the state space generated from the $\mu$ CRL specification by using the toolset CADP.

Table 1. One FS with capacity of 5

\begin{tabular}{|c|c|c|}
\hline Clients & States & Transitions \\
\hline 1 & 39 & 40 \\
2 & 413 & 456 \\
3 & 4381 & 5055 \\
4 & 4845 & 5681 \\
5 & 5309 & 6307 \\
\hline
\end{tabular}

Table 2. Three FSs with capacity of 1,2 and 3 respectively

\begin{tabular}{|c|c|c|}
\hline Clients & States & Transitions \\
\hline 1 & 49 & 50 \\
2 & 867 & 932 \\
3 & 12307 & 14073 \\
4 & 13449 & 15917 \\
5 & 14591 & 17761 \\
\hline
\end{tabular}

We first construct a system where only one FS is applied. The FS' capacity is set to 5 . A number of clients simultaneously request a phone connection. Before the simulation starts, all clients have preset enough money in their account. We incrementally increase the number of clients from 1 to 5 , and Table 1 illustrates the changes of the state space that result. It can be seen that when the second and third client are connected to the FS, the state space increases rapidly: by a factor of almost 10 . However, after this the subsequent increases level off, and the size is increased by roughly $20 \%$ when one new client is added to the system.

The same phenomenon is noticed as well when we apply three FSs to the system. The server capacities are set to 1, 2 and 3 respectively, and the resultant state space is shown in Table 2 This seems to suggest that, with the number of clients being increased, the state space will fairly reach a saturated point where the state space is slowly increased by a stable pace. We are currently investigating whether this is a general phenomenon or one peculiar to this particular example.

\section{Conclusions and Future Work}

Verification is an important process in the development of Erlang applications. This paper contributes to the recent strand of work which has studied the verification of Erlang applications using the process algebra $\mu \mathrm{CRL}$. The basic methodology in this approach 
is for an Erlang application to be translated (abstracted) into a $\mu \mathrm{CRL}$ specification, upon which the standard model checker CADP can be applied.

The study of how best to translate Erlang into $\mu \mathrm{CRL}$ contains many open research issues and is still in its early stage. Recent results had shown how components such as supervisor, gen_server, gen_fsm and the Erlang timeout event could be translated into $\mu \mathrm{CRL}$, but the translation rules for each component had been evaluated independently. At FORTE'07 we defined the rules for translating gen_fsm into $\mu \mathrm{CRL}$, and evaluated the rules with two case studies. This was extended in [16] by defining the rules for coping with Erlang timeout events and evaluated the work with some case studies. The experimental results show quite a promising effect for system verification.

However, no work had investigated the translation rules in an application where the gen_server, supervisor, gen_fsm and timeout events were incorporated in an integrated system. This forces us to face a challenge. If we apply all rules in an integrated way, will these rules show the similar effects for system verification as they independently demonstrated? Moreover, will a state space explosion mean that effective verification is lost? These questions are important to us since we want to make sure (or at least be confident) that all the defined rules can work in an integrated way, or that this requirement could be achieved through modifying some rules before we looked at the translation of other OTP components (such as applications).

In this paper, we have attempted to look at these questions by investigating the verification of an Erlang telecommunication system in $\mu \mathrm{CRL}$. The system integrates the use of the supervisor, gen_server, gen_fsm components and uses explicit timeout events. We have shown how the system is translated into a $\mu \mathrm{CRL}$ specification using the proposed translation rules, and verified a number of system properties by using CADP and investigated the changes of state space when the number of clients increases. In our experiments, a property under verification (PUV) is defined in a way where the behaviour of the functional servers (FSs), the behavior of the clients and the communication between the FSs and the clients should be verified simultaneously. Thus, each PUV looks at a property in the view of complete system. A faulty implementation was also used to test the capability of fault detection, and based upon the design the faulty implementation was correctly distinguished by CADP.

The experimental results suggest that when being applied in an integrated system, the translation rules show the similar effect for system verification as being applied independently. The study of the changes in state space suggests, with the number of clients being increased, the state space is slowly increased by a stable pace. All experimental evidence gives us confidence that we are working in the correct direction, and thus we can continue with the study of some other OTP components.

There remains much to be done. Work continues on the automation of the translation of additional OTP components, as does work on verifying the correctness of the translation against the Erlang semantics 10[11. This latter aspect remains a challenging task, for the full semantics for distributed nodes in an Erlang application can have, semantically, some very subtle behaviour, as discussed in, for example, 18.

\section{Acknowledgements}

This work is supported by the UK Engineering and Physical Sciences Research Council (EPSRC) grant EP/C525000/1. We would like to thank the developers of the tool sets of $\mu \mathrm{CRL}$ and CADP for permitting the use of tools for system verification. 


\section{References}

1. Armstrong, J., Virding, R., Wikström, C., Williams, M.: Concurrent Programming in Erlang, 2nd edn. Prentice-Hall, Englewood Cliffs (1996)

2. Arts, T., Benac-Earle, C., Derrick, J.: Verifying Erlang code: a resource locker case-study. In: Eriksson, L.-H., Lindsay, P.A. (eds.) FME 2002. LNCS, vol. 2391, pp. 184-203. Springer, Heidelberg (2002)

3. Arts, T., Benac-Earle, C., Penas, J.J.S.: Translating Erlang to $\mu$ CRL. In: The Fourth International Conference on Application of Concurrency to System Design (ACSD 2004), June 2004, pp. 135-144. IEEE Computer Society, Los Alamitos (2004)

4. Baeten, J.C.M., Weijland, W.P.: Process Algebra. Cambridge University Press, Cambridge (1990)

5. Benac-Earle, C.: Model checking the interaction of Erlang components. PhD thesis, The University of Kent, Canterbury, Department of Computer Science (2006)

6. Benac-Earle, C., Fredlund, L.-Å.: Verification of Language Based Fault-Tolerance. In: Moreno Díaz, R., Pichler, F., Quesada Arencibia, A. (eds.) EUROCAST 2005. LNCS, vol. 3643, pp. 140-149. Springer, Heidelberg (2005)

7. Benac-Earle, C., Fredlund, L.-Å., Derrick, J.: Verifying Fault-Tolerant Erlang Programs. In: Sagonas, K., Armstrong, J. (eds.) Proceedings of ACM SigPlan Erlang 2005 Workshop, pp. 26-34. ACM Press, New York (2005)

8. CADP, http://www.inrialpes.fr/vasy/cadp/

9. Clarke, E., Grumberg, O., Long, D.: Model Checking. MIT Press, Cambridge (1999)

10. Fredlund, L.Å: Towards a sematics for Erlang. In: Foundatins of Mobile Computation: A Post-Conference Satellite Workshop of FST and TCS (1999)

11. Fredlund, L.-Å.: A Framework for Reasoning about Erlang Code. PhD thesis, Roral Institute of Technology, Stockholm, Sweden (2001)

12. Fredlund, L.-A., Gurov, D., Noll, T., Dam, M., Arts, T., Chugunov, G.: A verification tool for Erlang. International Journal on Software Tools for Technology Transfer 4(4), 405-420 (2003)

13. Groote, J.F., Ponse, A.: The syntax and sematics of $\mu$ CRL. In: Algebra of Communicating Processes 1994, Workshop in Computing, pp. 26-62 (1995)

14. Guo, Q.: Verifying Erlang/OTP Components in $\mu$ CRL. In: Derrick, J., Vain, J. (eds.) FORTE 2007. LNCS, vol. 4574. pp. 227-246. Springer, Heidelberg (2007)

15. Guo, Q., Derrick, J.: Eliminating overlapping of pattern matching when verifying Erlang programs in $\mu$ CRL. In: Sha, E., Han, S.-K., Xu, C.-Z., Kim, M.-H., Yang, L.T., Xiao, B. (eds.) EUC 2006. LNCS, vol. 4096, Springer, Heidelberg (2006)

16. Guo, Q., Derrick, J.: Verification of Timed Erlang/OTP Components Using the Process Algebra $\mu$ CRL. In: Thompson, S., Fredlund, L.-A. (eds.) 6th ACM SIGPLAN Erlang Workshop, pp. 55-64. ACM Press, New York (2007)

17. Huch, F.: Verification of Erlang programs using abstract interpretation and model checking. ACM SIGPLAN Notices 34(9), 261-272 (1999)

18. Svensson, H., Fredlund, L.-AAA.: A More Accurate Semantics for Distributed Erlang. In: Thompson, S., Fredlund, L.-A. (eds) 6th ACM SIGPLAN Erlang Workshop, pp. 43-54. ACM Press, New York (2007) 